\title{
Factors regulating the expression of vascular permeability/ vascular endothelial growth factor by human vascular tissues
}

\author{
B.Williams \\ Department of Medicine, Leicester University School of Medicine, Leicester, UK
}

Diabetes mellitus is characterised by two important manifestations of endothelial dysfunction: 1) increased endothelial permeability to circulating macromolecules, e.g. albumin, and 2) endothelial proliferation leading to neovascularisation. We and others have proposed that a recently described cytokine family known as either vascular permeability factor (VPF) or vascular endothelial growth factor (VEGF) may play a key role in the development of the aforementioned manifestations of endothelial dysfunction in diabetes $[1,2]$. VPF/VEGF is an attractive candidate in this regard. VPF/VEGF dramatically increases vascular endothelial permeability and is established as the most potent, naturally occurring vascular permeabilising factor thus far identified. In addition, VPF/VEGF is a powerful mitogen for vascular endothelial cells and is recognised as an important independent stimulus for physiological and pathological angiogenesis [3,4]. The five closely related members of the VPF/VEGF family thus far identified comprise 24-42 kDa homodimeric, heparin binding glycoproteins (121, 145, 165, 189, 206 amino acids) produced by a variety of cells of human origin, including vascular smooth muscle cells (VSM). VPF/ VEGF $_{165}$ is the most abundant product of the human gene and is readily detectable in human serum [3-5].

VPF/VEGF binds with high affinity to the vascular endothelium via two specific receptor tyrosine kinases: the flt- 1 receptor and the KDR receptor [3, 4, $6]$. These receptors are almost exclusively expressed by vascular endothelium [6]. The regulation of VPF/ VEGF receptor expression in different tissues is

Corresponding author: Professor B. Williams, MD FRCP, Department of Medicine, Clinical Sciences Building, Leicester Royal Infirmary, PO Box 65, Leicester, LE2 7LX, UK

A bbreviations: VPF, Vascular permeability factor; VEGF, vascular endothelial growth factor; VSM, vascular smooth muscle cells; PKC, protein kinase C. poorly understood and it is not known whether specific VPF/VEGF functions, i.e. permeability or angiogenesis, are preferentially transduced by a specific receptor subtype. Moreover, understanding signal transduction via the VPF/VEGF receptors is limited, although VPF/VEGF mediated increases in microvascular permeability appear to be calcium dependent.

\section{VPF/NE GF and vascular disease in diabetes}

Support for the hypothesis that VPF/VEGF may play a role in the pathogenesis of diabetic microvascular complications comes from various recent observations. VPF/VEGF was detected in higher concentrations in the vitreous fluid of patients with active proliferative retinopathy when compared to those without evidence of neovascularisation [2]. Although this observation does not establish causality, further studies in which VPF/VEGF activity has been attenuated support the hypothesis that VPF/VEGF can play a key role in the pathogenesis of retinal neovascularisation. For example, Aiello et al. [7] have engineered and used soluble chimeric proteins that bind to free VPF/VEGF and limit its access to its receptors. The use of these "inhibitors" has been shown to suppress retinal neovascularisation in the mouse in vivo. Moreover, when antisense oligonucleotides designed to inhibit local VPF/VEGF synthesis were injected into the eyes of experimental animals with diabetes, the development of proliferative retinopathy was markedly attenuated. With regard to vascular permeability, Williamson et al. [8] recently showed that the acute infusion of VPF/VEGF into experimental animals markedly increased sciatic nerve and aortic albumin permeation. Furthermore, the topical application of high glucose concentrations $(30 \mathrm{mmol} / \mathrm{l})$ to a skin chamber in the rat in vivo, markedly increased 
albumin permeability across skin chamber vessels and this effect of high glucose was negated by the simultaneous application of a VPF/VEGF neutralising antibody. Evidence is thus accumulating to support the hypothesis that increased VPF/VEGF production may play a role in the pathogenesis of neovascularisation and the increased vascular permeability that characterises diabetic microangiopathy.

Increased vascular permeability within arteries is an important early manifestation of endothelial dysfunction in the pathogenesis of atherosclerosis [9]. It is tempting to speculate that production of VPF/ VEGF by human VSM cells within blood vessels may allow VPF/VEGF to act as a paracrine factor to influence the function, growth and permeability of the overlying vascular endothelium. It also seems likely that VPF/VEGF could play a key role in the regulation of glomerular permeability to protein. The kidney, in particular the glomerulus, is an abundant source of VPF/VEGF in man [10]. Our in situ hybridisation studies suggest that within the glomerulus VPF/VEGF mRNA is predominantly expressed by the glomerular epithelial cell where it is strategically placed to influence the permeability of the glomerular endothelium. Although a role for VPF/ VEGF in the regulation of glomerular permeability requires confirmation, acute infusions of $\mathrm{VPF} /$ VEGF into the rat markedly increase urinary protein excretion [11] suggesting that increased VPF/VEGF production (either renal or systemic) could potentially play a role in the pathophysiology of microalbuminuria and proteinuria in diabetic subjects.

\section{Factors regulating VPF/VE G F production}

VPF/VEGF mRNA has been detected in a variety of tissues including; lung, kidney, heart, adrenal gland, pituitary gland, brain, liver, spleen, gastric mucosa, breast and reproductive tissues [3, 4]. We have used cultured human VSM to study the factors regulating VPF/VEGF production. Human VSM cells predominantly express a single $4.2 \mathrm{~kb}$ mRNA transcript corresponding to the $\mathrm{VPF} / \mathrm{VEGF}_{165}$ peptide which is secreted by human VSM cells $[1,12]$. Using in situ hybridisation we have confirmed that there are low levels of constitutive VPF/VEGF mRNA in medium to large human arteries in vivo and that this is restricted to the VSM cells. Various stimuli have been shown to increase VPF/VEGF expression and production by cultured VSM cells including; hypoxia, vasopressor hormones such as angiotensin II and vasopressin, cytokines such as transforming growth factor $\beta$, interleukin-1, and growth factors such as fetal calf serum, fibroblast growth factor and platelet derived growth factor $[1,5,12-16]$. Moreover, many of these factors appear to act synergistically to enhance VPF/VEGF expression. Any or all of these factors could act as stimuli for VPF/VEGF synthesis in various tissues in diabetic subjects. We have recently evaluated two additional potentially important factors, notably high glucose concentrations and chronic cyclical mechanical strain.

Elevated glucose concentrations ( $20 \mathrm{mmol} / \mathrm{l})$ markedly increase VPF/VEGF mRNA expression and peptide secretion by human VSM cells (a threefold increase compared to glucose $5 \mathrm{mmol} / \mathrm{l}$ ) [17]. This effect is observed within $3 \mathrm{~h}$ of exposure to high glucose levels and is rapidly reversible after normalising the extracellular glucose concentration. Elevated glucose concentrations activate protein kinase $\mathrm{C}$ (PKC) in human VSM cells $[18,19]$ and PKC is important in the regulation of VPF/VEGF mRNA expression in response to many of the aforementioned stimuli $[5,13]$. Inhibition of glucose-induced PKC activation prevents the glucose-induced increase in VPF/VEGF mRNA expression and peptide production by human VSM cells [17]. These results demonstrate that elevated glucose concentrations per se are a potent stimulus for VPF/VEGF production by human vascular cells and that the intracellular signalling pathway involves glucose-induced PKC activation.

Diabetes is frequently associated with hypertension and the latter has been strongly implicated in the pathogenesis of endothelial dysfunction, macrovascular and microvascular disease. In an endeavour to mimic the increased mechanical forces of hypertension, we have exposed cultured human VSM cells to chronic cyclical mechanical strain. When compared to static human VSM cells, the imposition of cyclical mechanical strain significantly increased VPF/VEGF mRNA expression and peptide production [20]. Moreover, the rate of VPF/VEGF production was proportional to the magnitude of mechanical strain applied. These observations suggest that the mechanical stress imposed on blood vessels and other tissues in hypertensive subjects may also provide an important stimulus for VPF/VEGF production.

\section{Conclusions}

There is ample circumstantial evidence to implicate $\mathrm{VPF} / \mathrm{VEGF}$ in the pathogenesis of the endothelial dysfunction, permeability defect and neovascularisation that characterise diabetes. Moreover, there are numerous stimuli, including glucose itself, that could potentially interact to increase VPF/VEGF production in diabetic subjects. An improved understanding of the cellular regulation of VPF/VEGF production and flt- 1 and KDR receptor expression and function may ultimately yield novel and potent adjunctive therapies to limit the microvascular complications of diabetes. 


\section{References}

1. Williams B, Quinn-Baker A, Gallacher B, Lodwick D (1995) Angiotensin II increases vascular permeability factor gene expression by human vascular smooth muscle cells. Hypertension 25: 913-917

2. Aiello LP, Avery R, Arrig P et al. (1994) Vascular endothelial growth factor in ocular fluid of patients with diabetic retinopathy and other retinal disorders. N Engl J Med 331: 1480-1487

3. Ferrara N, Houck K, Jakeman L, Leung DW (1992) Molecular and biological properties of the vascular endothelial growth factor family of proteins. Endocr Rev 13: 18-32

4. Senger DR, Van Der Water L, Brown LF (1993) Vascular permeability factor in tumor biology. Cancer Metastasis Rev 12: 303-324

5. Williams B (1996) Vascular permeability/vascular endothelial growth factors: a potential role in the pathogenesis and treatment of vascular diseases. Vasc Med 1: 251-258

6. Jakeman LB, Winer J, Bennet GL, Altar CA, Ferrara N (1992) Binding sites for vascular endothelial growth factor are localized on endothelial cells in adult rat tissues. J Clin Invest 89: 244-253

7. Aeillo LP, Pierce EA, Foley ED et al. (1995) Suppression of retinal neovascularization in vivo by inhibition of vascular endothelial growth factor (VEGF) using soluble VEGF-receptor chimeric proteins. Proc Natl Acad Sci USA 92: 10457-10461

8. Williamson JR, Chang KC, Stephan CC, Brock TA, Tilton RG (1996) Links between neural and aortic vascular dysfunction by elevated glucose levels and VEGF. Diabetes (1996) 45: [Suppl 2] p 66A (Abstract 237)

9. Ross R (1993) The pathogenesis of atherosclerosis: a perspective for the 1990s. Nature 362: 801-809

10. Simon M, Grone H-J, Johren O, Plate KH, Fuchs E, Risau W (1995) Expression of vascular endothelial growth factor and its receptors in human renal ontogenesis and in adult kidney. Am J Physiol 268: F240-F250
11. Iijima K, Yoshikawa N, Connolly DT (1992) Expression of vascular permeability factor by mesangial cells. J Am Soc Nephrol 3: 514 (Abstract)

12. Williams B, Quinn-Baker A, Gallacher B (1995) Serum and platelet-derived growth factor-induced vascular permeability factor mRNA expression by human vascular smooth muscle cells in vitro. Clin Sci 88: 141-147

13. Williams B, Gallacher B, Patel H, Orme C (1995) The potential role of vasopressin as a modulator of endothelial function. In: Saito T, Kurokawa K, Yoshida S (eds) Neurohypophysis: Recent progress of vasopressin and oxytocin research. Elsevier Science B. V. Amsterdam, pp 573-582

14. Brogi E, Wu T, Namiki A, Isner JM (1994) Indirect angiogenic cytokines upregulate VEGF and bFGF gene expression in vascular smooth muscle cells, whereas hypoxia upregulates VEGF expression only. Circulation 90: 649-652

15. Stavri GT, Zachary IC, Baskerville PA, Martin JF, Erusalimsky JD (1995) Basic fibroblast growth factor upregulates the expression of vascular endothelial growth factor in vascular smooth muscle cells: synergistic interaction with hypoxia. Circulation 92: 11-14

16. Shweiki D, Itin A, Soffer D, Keshet E (1992) Vascular endothelial growth factor induced by hypoxia may mediate hypoxia-initiated angiogenesis. Nature 359: 843-845

17. Gallacher B, Patel HR, Williams B (1996) Glucose induced increases in vascular permeability factor mRNA and peptide production by human vascular smooth muscle cells. J Hypertension 14: S84

18. Williams B, Schrier RW (1992) Characterization of glucose-induced in situ protein kinase $\mathrm{C}$ activity in cultured vascular smooth muscle cells. Diabetes 41: 1464-1472

19. Williams B (1995) Glucose-induced vascular smooth muscle dysfunction: the role of protein kinase C. J Hypertension 13: 477-486

20. Kimber P, O'Callaghan CJ, Williams B (1996) Chronic cyclical stretch increases production of vascular permeability factor mRNA and peptide in human vascular smooth muscle cells. J Hypertension 14: S66 